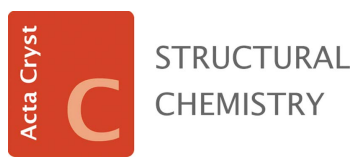

ISSN 2053-2296
Keywords: book review; Raman spectroscopy

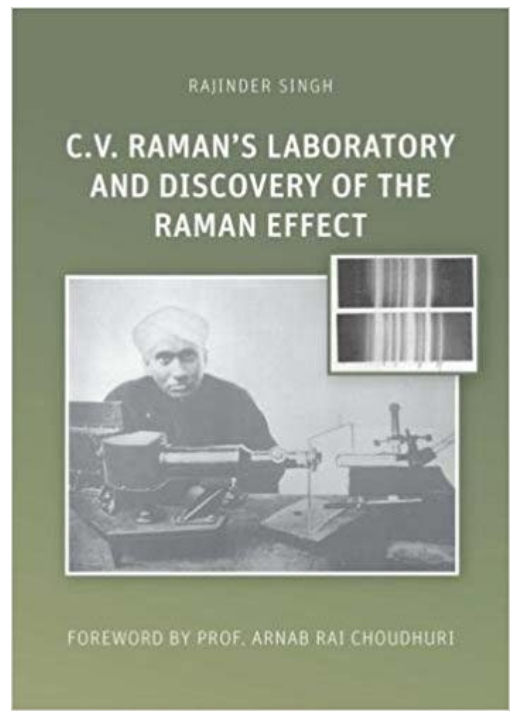

(C) 2018 International Union of Crystallography

\section{C.V. Raman's Laboratory and Discovery of the Raman Effect. By Rajinder Singh. Shaker Verlag, 2018. Paperback, 172 pages. Price EUR 21.90/SFr 27.40. ISBN 978-3-8440-5691-4 \\ Boriana Mihailova*}

University of Hamburg, Department of Earth Sciences, Grindelallee 48, D-20146 Hamburg, Germany. *Correspondence e-mail: boriana.mihailova@uni-hamburg.de

The common things that pop into our minds when we hear 'Raman' are 'Raman spectroscopy', 'atomic dynamics', 'local structure', etc. Very seldom do we think about the person, the brilliant Indian scientist Chandrasekhara Venkata Raman, who discovered and reported for the first time the phenomenon of inelastic light scattering by atomic vibrations, subsequently named after him.

This book would be of interest for everyone who would like to learn about the discovery of the Raman effect. However, to a certain extent, this is an unusual biography. Scientists or students who know only a little about C.V. Raman might be slightly disappointed, because the book is more about C.V. Raman as a politician than as a person and scientist; it is more about the political atmosphere at the University of Calcutta at that time than about the research activities in the group of C.V. Raman that led to the observation of a new type of secondary radiation which is nowadays routinely applied in modern materials science. On the other hand, readers who are more or less familiar with the biography of C.V. Raman will appreciate this atypical approach in telling the story of this epochal breakthrough in physics, which was the missing experimental proof everyone was looking for, to boost the modern conception of the nature of light.

Actually, this was the goal of the author, Dr Rajinder Singh, namely to present facts 'from the kitchen', which are not so popular among the canonical admirers of C.V. Raman. For example, it is curious to see that in a biography of Raman, it is emphasized that there were several quite incorrect statements in the original paper [C. V. Raman \& K. S. Krishnan (1928). Nature, 121, 501-502]. The frank presentation of the facts by Dr Singh does not belittle the efforts and the genius of C.V. Raman; on the contrary, it demonstrates the insight of C.V. Raman in immediately recognising that the search for inelastic visible-light scattering had finally been successful, and his courage in publishing the results as soon as possible in order to make the new findings available for the scientific community worldwide. The book provides technical details about the instruments used to discover the Raman effect as well as about the funds available for research at the University of Calcutta. Furthermore, this short monograph can be considered as a nice overview of the history of Indian physical science in that amazing era of the revolutionary development of modern physics, which should be of interest for an international readership. 